\title{
Prevalência e fatores associados à autopercepção corporal em escolares do nordeste brasileiro
}

\author{
Dixis Figueroa Pedraza ${ }^{1}$ \\ Carolina Pereira da Cunha Sousa ${ }^{2}$ \\ Ricardo Alves de Olinda ${ }^{3}$
}

\section{RESUMO}

Objetivou-se avaliar a autopercepção corporal em escolares da rede pública de ensino de Campina Grande e examinar a associação com o sexo, a idade e o estado nutricional. Estudo transversal com 1081 crianças de cinco a dez anos. As crianças foram pesadas e medidas segundo procedimentos padronizados. O estado nutricional foi obtido por meio do IMC/I. Para a avaliação da percepção corporal utilizou-se a escala Children's Body Image Scale. A prevalência de sobrepeso foi de 75,9\%. Crianças com sobrepeso e obesas apresentaram maiores percentuais de insatisfação corporal. Das crianças obesas, 73,7\% perceberam-se mais magras. Constatou-se que $94,7 \%$ dos escolares com sobrepeso, $98 \%$ dos obesos e $41,8 \%$ dos eutróficos gostariam de ser mais magros, enquanto $83,3 \%$ dos desnutridos desejariam ser mais gordos. A visão mais distorcida do corpo verificou-se nas crianças mais velhas e nos meninos. Aponta-se que o sexo, a idade e o estado nutricional podem influenciar a percepção corporal.

Descritores: Imagem Corporal; Peso Corporal; Saúde da Criança.

\footnotetext{
${ }^{1}$ Bacharel em Alimentos, Doutor em Nutrição. Professor Doutor da Universidade Estadual da Paraíba. Campina Grande, PB, Brasil. E-mail: dixisfigueroa@gmail.com.

${ }^{2}$ Enfermeira, Mestre em Saúde Pública. Professora Substituta da Universidade Federal de Campina Grande. Campina Grande, PB, Brasil. E-mail: carolina_pcs@hotmail.com.

${ }^{3}$ Estatístico, Doutor em Estatística e Experimentação Agronômica. Professor Adjunto C da Universidade Estadual da Paraíba. Campina Grande, PB, Brasil. E-mail: ricardo.estat@yahoo.com.br.
}

Artigo recebido: 01/11/2016.

Artigo aprovado: 26/10/2017.

Artigo publicado: 16/04/2018.

\section{Como citar esse artigo:}

Pedraza DF, Sousa CPC, Olinda RA. Prevalência e fatores associados à autopercepção corporal em escolares do nordeste brasileiro. Rev. Eletr. Enf. [Internet]. 2018 [acesso em: ];20:v20a02. Disponível em: http://doi.org/10.5216/ree.v20.43937. 


\section{INTRODUÇÃO}

A imagem corporal refere-se a um construto multifatorial que envolve a percepção, os pensamentos, os sentimentos, as atitudes e os comportamentos relacionados a si próprio e às outras pessoas ${ }^{(1)}$. A construção dessa percepção se relaciona às crenças, desejos, emoções e comportamentos em relação a si próprio e às outras pessoas. Compondo a imagem corporal, a satisfação e a autopercepção são fatores preponderantes na autoaceitação dos indivíduos e podem gerar situações de insatisfação (diferenças entre o corpo percebido e o ideal) e discrepâncias perceptuais (superestimação ou subestimação de toda a imagem corporal ou de partes dela $)^{(1-3)}$.

Desde a infância, a imagem corporal é um importante componente da autoestima e da relação que envolve a percepção do próprio corpo somada à experiência de si mesmo ${ }^{(4)}$. Nesse período da vida, o ganho de peso é proporcionalmente maior que o aumento da estatura, uma vez que ocorre o fenômeno da repleção energética ou puberal, em que meninos e meninas apresentam maior velocidade de crescimento de tecido adiposo como forma de reserva energética para ser usado na fase de intenso crescimento pubertário que ocorre na adolescência ${ }^{(5)}$. A associação deste fenômeno biológico ao sedentarismo, comportamento que também se inicia nesse período ${ }^{(5)}$, torna a infância um estágio biologicamente vulnerável a distúrbios nutricionais e, consequentemente, sugestiva à distorção perceptiva e à insatisfação corporal(4-5).

Apesar dessa vulnerabilidade, a imagem corporal advinda da infância não é determinística, como acontece na adolescência, mas apriorística, pois caracteriza o lugar do sujeito no espaço, propiciando a formação de uma identidade mediada pelo contato entre o próprio sujeito e o mundo. Essa condição favorece a construção de uma nova imagem corporal obtida por meio de vivências e não de padrões estereotipados ${ }^{(6)}$. Entretanto, as crianças têm sido alvo de poucos estudos relacionados ao tema, sendo os mesmos desenvolvidos principalmente nos Estados Unidos, Austrália e Reino Unido. Os resultados sistematizados indicam altas prevalências de insatisfação corporal e de percepção distorcida da imagem ${ }^{(7-8)}$.

Abordar a percepção da imagem corporal na infância revela-se essencial para a prevenção e minimização dos efeitos gerados pela promoção do corpo magro ideal imposto pelas sociedades ocidentais ${ }^{(9)}$. Nesse sentido, 0 ambiente escolar destaca-se por possibilitar o planejamento integrado de ações preventivas junto aos setores da saúde e educação(10). Ainda, as escolas podem constituir espaços privilegiados para promover mudanças nos conceitos de imagem corporal, conscientizar sobre os prejuízos que as condutas para redução de peso pode desencadear na saúde, bem como na orientação sobre hábitos alimentares mais saudáveis ${ }^{(9)}$.

Face ao exposto, o presente estudo pretende avaliar a autopercepção corporal em crianças escolares que frequentam a rede pública de ensino do município de Campina Grande e examinar a associação com o sexo, a idade e o estado nutricional.

\section{MÉTODOS}

Estudo transversal realizado com escolares de Campina Grande, Paraíba/Brasil. Campina Grande localizase na mesorregião do agreste paraibano e possui área total de $641 \mathrm{Km}^{2}$. A cidade apresenta populações urbana e rural com 367.278 e 17.998 habitantes, respectivamente. Possui 51 bairros e cinco distritos, sendo três rurais 
(Galante, São José da Mata e Catolé de Boa Vista) e dois urbanos (Catolé de Zé Ferreira e Santa Terezinha). Os bairros de Campina Grande estão delimitados em cinco regiões: centro, norte, leste, sul e oeste.

No período da coleta de dados (setembro de 2013), o município contava com 120 escolas de ensino

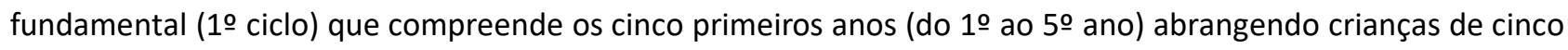
a 10 anos de idade. Localizavam-se 86 escolas na zona urbana (nove núcleos) e 34 na zona rural (quatro núcleos), que atendiam, respectivamente, 21.696 e 2.801 escolares. A população elegível para participar do estudo incluiu todas as crianças escolares, na faixa etária de cinco a 10 anos, de escolas urbanas da rede pública de ensino do município. Optou-se por trabalhar com crianças escolares devido aos poucos estudos com esse grupo populacional no Brasil e pelo fato do ambiente escolar oportunizar a adoção de medidas preventivas e o diálogo entre profissionais das áreas de educação e saúde. Crianças da zona rural e do ensino privado do município não foram consideradas por representarem uma parcela muito pequena do grupo de interesse.

Para garantir a representatividade das regiões e das diversidades administrativas, as escolas foram ordenadas para sorteio segundo a distribuição por núcleos adotados pelo município. Em cada núcleo da zona urbana foram sorteadas duas escolas, totalizando 18. Em cada escola foi sorteada uma turma de cada ano, correspondente a 90 turmas. Todas as crianças das turmas sorteadas que frequentavam a escola no dia da coleta de dados foram consideradas elegíveis para o estudo, representando 1.754 escolares (342 do primeiro ano, 341 do segundo ano, 348 do terceiro ano, 346 do quarto ano e 377 do quinto ano). Desses, foram excluídos os escolares que estavam fora da faixa etária (escolares do primeiro ano e do quinto ano menores de cinco anos e maiores de dez anos) e aqueles com problemas físicos que dificultassem a avaliação antropométrica. Houve perdas devido a escolares que não estavam presentes na escola no dia da coleta de dados e por dificuldades na avaliação antropométrica. Assim, do total de 1.754 escolares, foram analisados 1.093 (251 do primeiro ano, 261 do segundo ano, 243 do terceiro ano, 204 do quarto ano e 134 do quinto ano). Ainda, foram excluídos do banco de dados 12 questionários por incompletude das informações, totalizando 1.081 escolares de cinco a 10 anos de idade para a análise dos dados, sendo 245 do primeiro ano, 258 do segundo ano, 242 do terceiro ano, 204 do quarto ano e 132 do quinto ano. O fluxograma da seleção dos sujeitos da pesquisa é apresentado na Figura 1.

Os dados antropométricos foram coletados por uma equipe de alunos de graduação e pós-graduação da área de saúde da Universidade Estadual da Paraíba. Os alunos foram treinados para a padronização das medidas e da coleta de dados por meio de questionário. Foi elaborado um manual de orientação para as medições da estatura e do peso seguindo as recomendações técnicas internacionais ${ }^{(11)}$. A percepção corporal foi avaliada com a utilização da escala validada Children's Body Image Scale ${ }^{(12)}$.

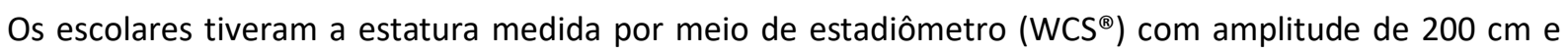
subdivisões de 0,1 cm. As medições foram realizadas em duplicata, aceitando-se variação máxima de 0,3 mm, e a medida final resultou da estimativa da média das duas medições. As crianças foram pesadas utilizando balança eletrônica do tipo plataforma com capacidade para 150 kg e graduação em 100g (Tanita UM-080®).

O estado nutricional das crianças foi estimado por meio do índice de massa corporal para idade (IMC/I) $\left(\mathrm{kg} / \mathrm{m}^{2}\right)$ e expresso em escore-Z de acordo com o padrão de referência do Multicentre Growth Study (Who Reference 5-19 years) ${ }^{(13)}$. Os cálculos foram realizados com a utilização do programa WHO AnthroPlus 2009. 
Escolares com IMC/I < Escore-z -2, $\geq$ Escore-z -2 e < Escore-z +1, > Escore-z +1 e < Escore-z +2, $\geq$ Escore-z + 2, foram classificados como desnutridos, eutróficos, com sobrepeso e obesos, respectivamente ${ }^{(14)}$.

Figura 1: Fluxograma de seleção da população do estudo sobre o estado nutricional e os hábitos alimentares de escolares de cinco a 10 anos de idade.

1754 escolares de 5 a 10 anos de idade

[primeiro ano: $342(19,5 \%)$, segundo ano: $341(19,4 \%)$, terceiro ano: $348(19,8 \%)$, quarto ano: $346(19,7 \%)$, quinto ano: $377(21,6 \%)]$

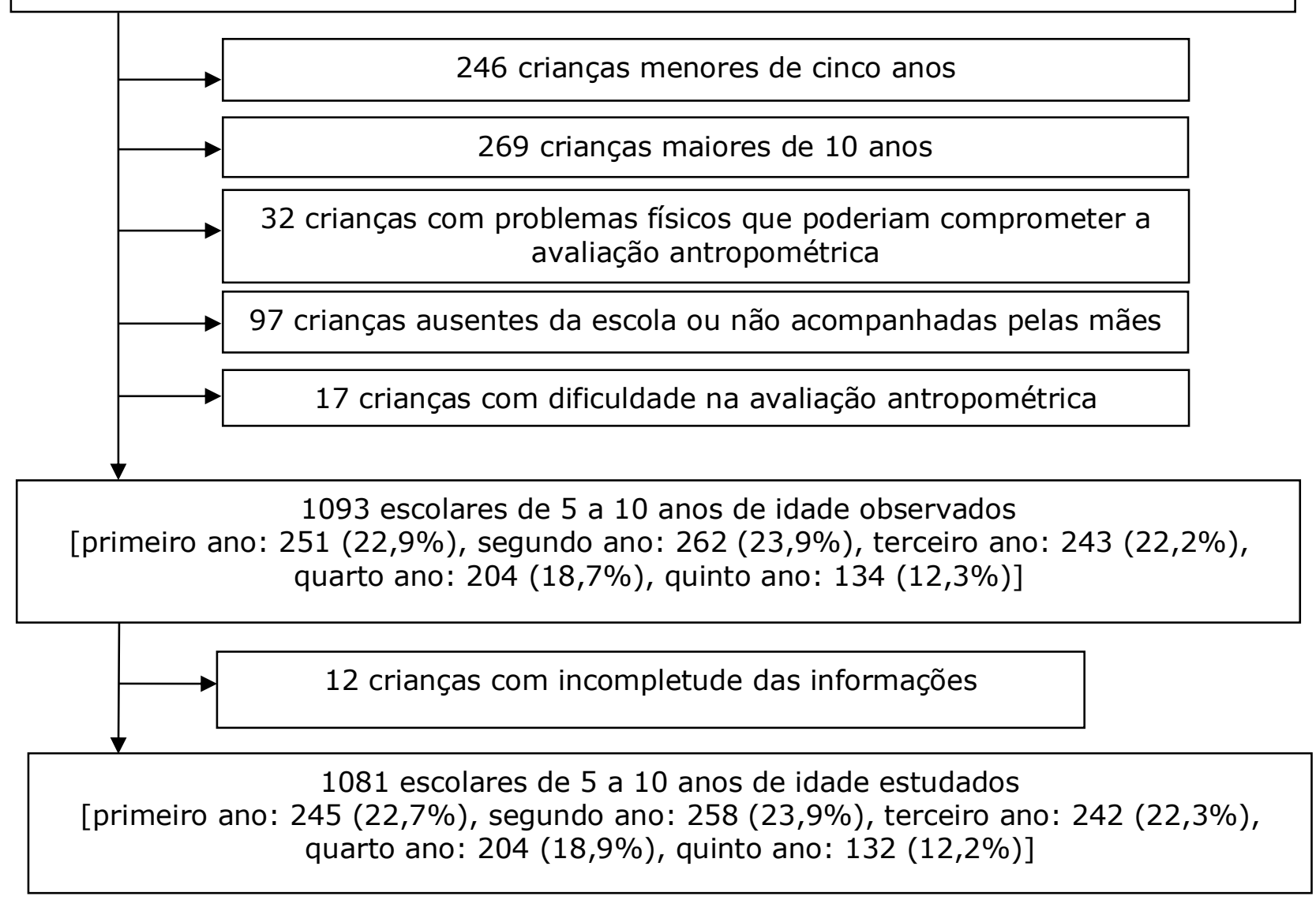

A escala Children's Body Image Scale ${ }^{(12)}$ foi utilizada conforme recomendações. Consiste de dois conjuntos de sete fotografias de acordo com o sexo da criança. Cada fotografia corresponde a um intervalo de $\mathrm{IMC}\left(\mathrm{kg} / \mathrm{m}^{2}\right)$. Inicialmente os escolares recebiam duas cópias da escala. Em seguida eram orientados a olhar a primeira escala, quando o entrevistador realizou a primeira pergunta: Que menino(a) tem o corpo mais parecido ao seu? (corpo percebido). Posteriormente, eram orientados a olhar a segunda escala, quando o entrevistador realizou a segunda pergunta: Que menino(a) tem o corpo que você gostaria de ter? (corpo idealizado).

O IMC/I diagnosticado foi categorizado de acordo com os intervalos de IMC sugeridos na escala. Definiu-se, assim, o corpo real do escolar considerando as categorias usadas na escala.

Para avaliar a percepção corporal dos escolares foram considerados três parâmetros:

I. visão corporal, que corresponde à comparação entre o corpo percebido e o corpo real. Diante disso, havia três situações:

1. o corpo percebido foi o mesmo que o idealizado, sendo assim o escolar "identifica-se igual a como é"; 
2. o corpo percebido foi menor que o real, sendo assim o escolar "identifica-se mais magro do que é";

3. o corpo percebido foi maior do que o real, sendo assim o escolar "identifica-se mais gordo do que é".

II. insatisfação corporal, que corresponde à comparação entre o corpo percebido e o corpo idealizado. Diante disso, havia três situações:

1. o corpo percebido foi o mesmo que o idealizado, sendo assim o escolar "identifica-se igual a como gostaria de ser";

2. o corpo percebido foi menor que o idealizado, sendo assim o escolar "identifica-se mais magro do que gostaria de ser";

3. o corpo percebido foi maior que o idealizado, sendo assim o escolar "identifica-se mais gordo do que gostaria de ser".

III. idealização corporal, que corresponde à comparação entre o corpo real e o corpo idealizado. Diante disso, havia três situações:

1. o corpo real foi o mesmo que o idealizado, sendo assim o escolar "é igual a como gostaria de ser";

2. o corpo real foi menor que o idealizado, sendo assim o escolar "é mais magro do que gostaria de ser";

3. o corpo real foi maior que o idealizado, sendo assim o escolar "é mais gordo do que gostaria de ser".

Para cada parâmetro foram calculadas as discrepâncias de percepção corporal: discrepância de visão corporal (diferença entre corpo percebido e o corpo real), discrepância de insatisfação corporal (diferença entre corpo percebido e o corpo idealizado) e discrepância de idealização corporal (diferença entre o corpo real e o corpo idealizado).

Para verificar as discrepâncias de visão corporal, insatisfação corporal e idealização corporal em função do sexo, utilizou-se o T-test. Diferenças em função da idade e do IMC/I identificaram-se por meio de ANOVA com provas post-hoc de Bonferroni. Todas as análises foram realizadas com o auxílio do software R. Foi considerada associação significante quando $p<5 \%$.

O projeto de pesquisa foi aprovado pelo Comitê de Ética em Pesquisa da Universidade Estadual da Paraíba sob o no 19690513.6.0000.5187. Todos os pais e/ou responsáveis pelas crianças assinaram o Termo de Consentimento Livre e Esclarecido. Os resultados foram divulgados para as escolas e Secretaria de Educação do município.

\section{RESULTADOS}

Do total de 1.081 crianças observadas, o sobrepeso foi diagnosticado em 12,3\% dos escolares e a obesidade em 9,2\%, conforme avaliação antropométrica.

Ao verificar a percepção corporal, observou-se que $83,8 \%$ das crianças com obesidade percebem o corpo de maneira contrária, destes, $73,7 \%$ percebem-se mais magras e 10,1\% mais gordas. Nas crianças com sobrepeso, 
a percepção corporal alterada foi constatada em $85,7 \%$, destes $75,9 \%$ perceberam-se mais magras e 9,8\% mais gordas. Entre as crianças desnutridas, 41,7\% perceberam-se mais gordas (Figura 2).

Figura 2: Distribuição das crianças segundo a visão corporal, comparando o corpo percebido em relação ao corpo real.

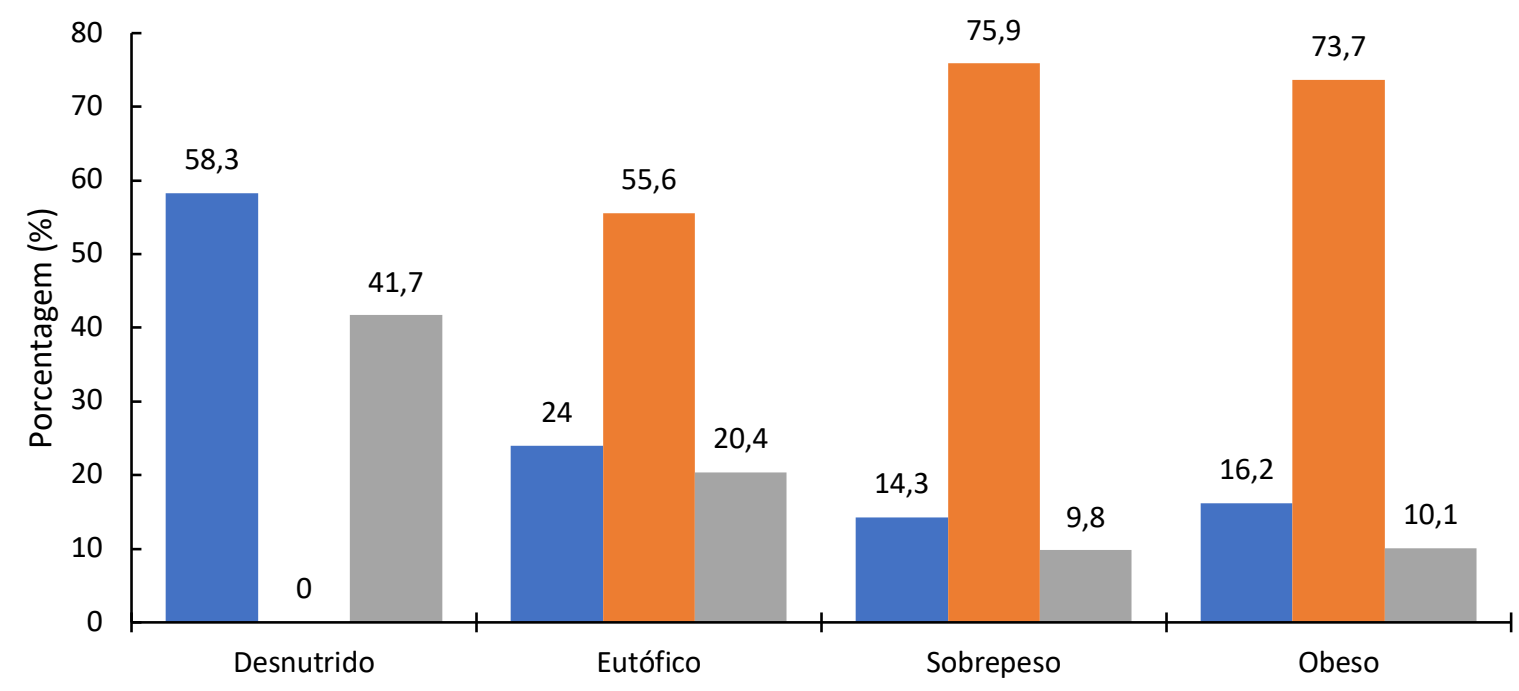

- Identifica-se igual a como é $\quad$ Identifica-se mais magro do que é Identifica-se mais gordo do que é

Quanto à insatisfação corporal, os resultados apontaram que 86,0\% dos escolares estavam insatisfeitos com seu corpo. Destes, $45 \%$ gostariam de ser mais magras e $41 \%$ gostariam de ser mais gordas (Figura 3 ).

Figura 3: Distribuição das crianças segundo a insatisfação corporal, comparando o corpo percebido em relação ao corpo idealizado.

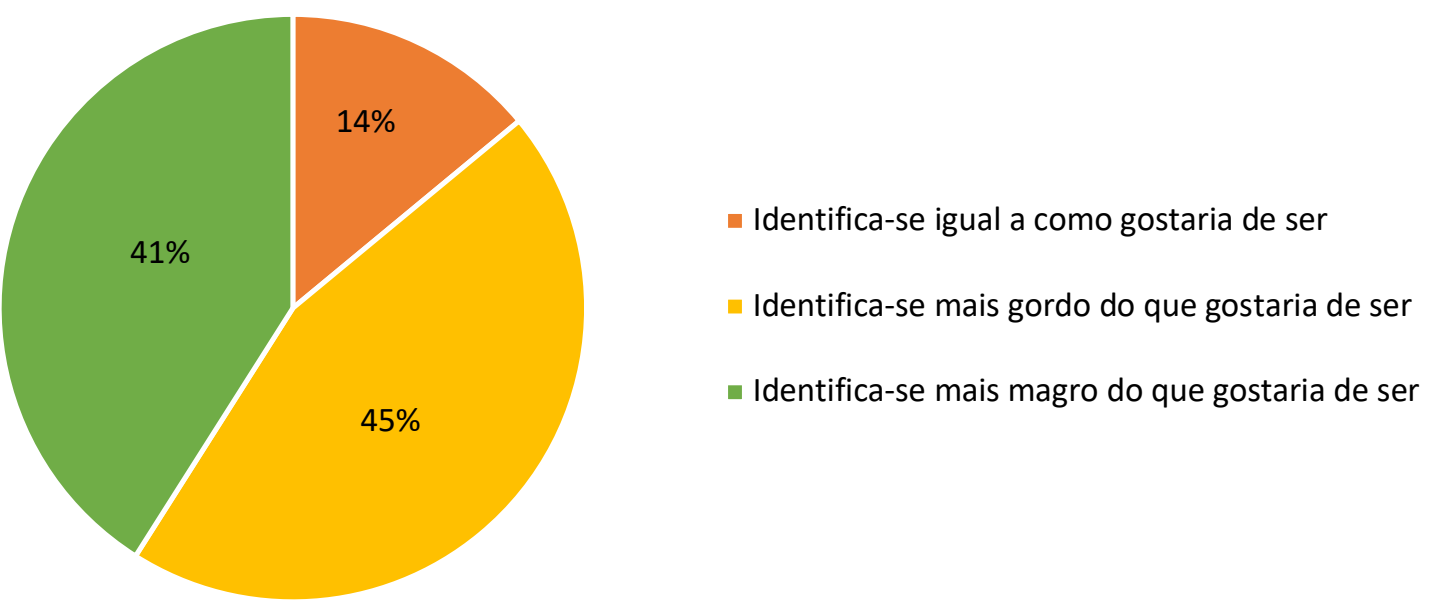

Considerando a idealização corporal, observou-se que $98,0 \%$ das crianças obesas, $94,7 \%$ das com sobrepeso e $45,8 \%$ das eutróficas gostariam de ser mais magras do que se apresentavam. Entre as crianças desnutridas, $83,3 \%$ gostariam de ser mais gordas (Figura 4 ). 
Figura 4: Distribuição das crianças segundo a idealização corporal, comparando o corpo real em relação ao corpo idealizado.

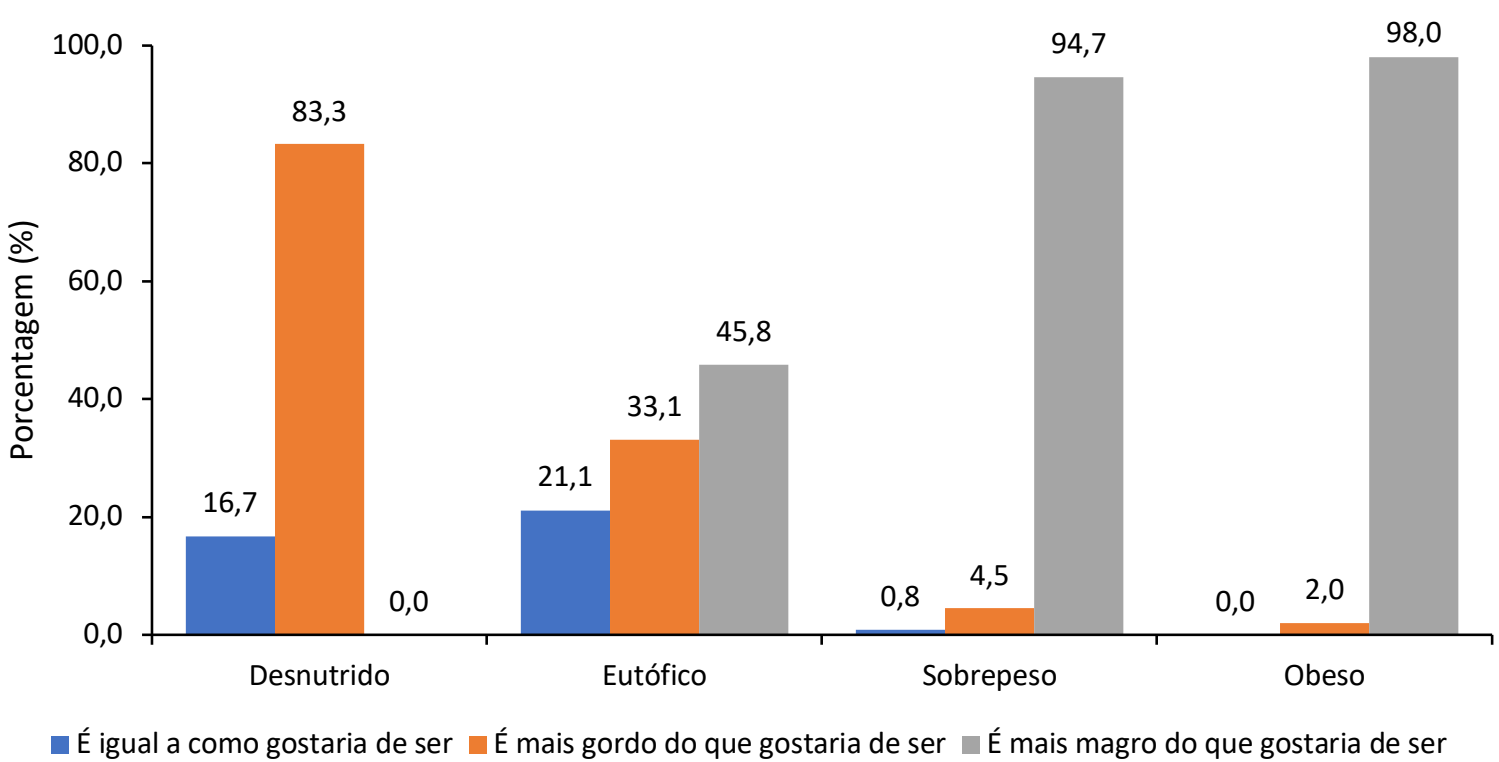

Na Tabela 1 são apresentados os resultados das análises das medidas de percepção corporal segundo idade, sexo e estado nutricional da criança. Observou-se visão corporal mais distorcida (mais magros) nas crianças de 99,11 anos e de 10-10,11 anos em relação aos menores de sete anos. Resultado similar obteve-se segundo o sexo com maior discrepância entre a imagem percebida e a real nos meninos. Para a insatisfação corporal, constatouse que crianças obesas e com sobrepeso apresentaram maiores médias de insatisfação corporal (mais gordas) quando comparadas aos outros grupos. Crianças com obesidade apresentaram, ainda, maior média de discrepância perceptiva do que aquelas com sobrepeso. Quanto à idealização do corpo (gostariam de ser mais magras do que são), observou-se que crianças com idades extremas (9-9,11 anos e 10-10,11 anos) apresentaram médias mais discrepantes. 
Pedraza DF, Sousa CPC, Olinda RA.

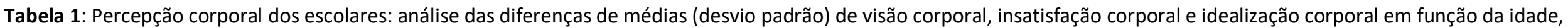
sexo e estado nutricional. Campina Grande, Paraíba, Brasil 2014.

\begin{tabular}{|c|c|c|c|c|c|c|c|c|c|c|c|c|c|c|c|}
\hline \multicolumn{16}{|c|}{ Visão Corporal (corpo percebido - corpo real) } \\
\hline \multicolumn{9}{|c|}{ Idade (anos) } & \multirow{2}{*}{$\mathbf{F}$} & \multirow{2}{*}{$p$} & \multicolumn{3}{|c|}{ Sexo } & \multirow{2}{*}{\multicolumn{2}{|c|}{ T-test }} \\
\hline \multicolumn{2}{|c|}{$<7$} & $7-7,11$ & \multicolumn{2}{|c|}{$8-8,11$} & \multicolumn{2}{|c|}{$9-9,11$} & \multicolumn{2}{|c|}{$10-10,11$} & & & Menina & & Menino & & \\
\hline \multicolumn{2}{|c|}{$-0,38(1,84)_{a}$} & $\begin{array}{c}-0,65 \\
(1,48)_{\text {ba }}\end{array}$ & \multicolumn{2}{|c|}{$-0,64(1,57)_{\mathrm{ba}}$} & $\begin{array}{r}-0, \\
(1,1\end{array}$ & $\begin{array}{l}92 \\
6)_{b c}\end{array}$ & \multicolumn{2}{|c|}{$-1,16(1,20)_{c}$} & 6,97 & $<0,001$ & \multicolumn{2}{|l|}{$-0,59(1,50)_{a}$} & $-0,81(1,54)_{b}$ & \multicolumn{2}{|l|}{0,02} \\
\hline \multicolumn{16}{|c|}{ Insatisfação Corporal (corpo percebido - corpo idealizado) } \\
\hline \multicolumn{5}{|c|}{ Idade (anos) } & \multirow{2}{*}{$\mathbf{F}$} & \multirow{2}{*}{$p$} & \multicolumn{4}{|c|}{ IMC/I } & \multirow{2}{*}{$\mathbf{F}$} & \multirow{2}{*}{$\boldsymbol{p}$} & \multicolumn{2}{|c|}{ Sexo } & \multirow{2}{*}{$\begin{array}{c}\mathrm{T}- \\
\text { test }\end{array}$} \\
\hline$<7$ & $7-7,11$ & $8-8,11$ & $9-9,11$ & $10-10,11$ & & & Desnutrido & Eutrófico & Sobrepeso & Obeso & & & Menina & Menino & \\
\hline $\begin{array}{c}-0,01 \\
(2,34)_{a}\end{array}$ & $\begin{array}{r}-0,22 \\
(2,29)_{a}\end{array}$ & $\begin{array}{c}0,05 \\
(2,08)_{a}\end{array}$ & $\begin{array}{c}0,12 \\
(1,86)_{a}\end{array}$ & $\begin{array}{c}0,14 \\
(1,86)_{a}\end{array}$ & 1,05 & 0,37 & $\begin{array}{r}-1,08 \\
(2,45)_{c}\end{array}$ & $\begin{array}{c}-0,44 \\
(1,93)_{c}\end{array}$ & $\begin{array}{c}1,25 \\
(2,00)_{b}\end{array}$ & $\begin{array}{c}2,23 \\
(1,65)_{a}\end{array}$ & 79,71 & $<0,001$ & $1 \quad 0,08(2,28)_{a}$ & $\begin{array}{c}-0,087 \\
(1,97)_{a}\end{array}$ & 0,19 \\
\hline \multicolumn{16}{|c|}{ Idealização Corporal (corpo real - corpo idealizado) } \\
\hline \multicolumn{9}{|c|}{ Idade (anos) } & \multirow{2}{*}{$\mathbf{F}$} & \multirow{2}{*}{$p$} & \multicolumn{3}{|c|}{ Sexo } & \multirow{2}{*}{\multicolumn{2}{|c|}{ T-test }} \\
\hline & & $7-7,11$ & & 11 & $9-c$ &, 11 & $10-1$ &, 11 & & & Menina & & Menino & & \\
\hline 0,37 & 36) $)_{b}$ & $\begin{array}{c}0,43 \\
(2,25)_{b}\end{array}$ & 0,70 & ,14) ba & $1,04($ & $2,14)_{a}$ & $1,30($ & ,94) $)_{a}$ & 6,07 & $<0,001$ & $0,67(2,33)_{a}$ & & $0,72(2,10)_{a}$ & 0,72 & \\
\hline
\end{tabular}

IMC/I: Índice de Massa Corporal para Idade $\left(\mathrm{kg} / \mathrm{m}^{2}\right)$; médias que compartilham subíndices iguais não são significativamente diferentes entre si $(p>0,05)$. 


\section{DISCUSSÃO}

O perfil antropométrico das crianças apontou prevalências de sobrepeso $(12,3 \%)$ e obesidade $(9,2 \%)$ inferiores às reportadas no nível nacional segundo a Pesquisa de Orçamentos Familiares de 2008-2009 que indicou taxas de $33,5 \%$ e $14,3 \%$, respectivamente ${ }^{(15)}$.

Entretanto, as taxas assemelham-se a estimativas de países em desenvolvimento que indicam prevalências de excesso de peso/obesidade de $12,9 \%$ entre crianças e adolescentes do sexo masculino e de $13,4 \%$ para o caso das meninas ${ }^{(16)}$. Apesar das diferenças ressaltadas nessas prevalências, as mesmas são expressivas de um problema de saúde pública que representa o momento epidemiológico de transição nutricional da população mundial e brasileira, inclusive o aumento de excesso de peso entre as crianças em idade escolar ${ }^{(16-17)}$. Nesse sentido, destaca-se o papel que a escola pode exercer na implementação de ações educativas e de prevenção(10,18).

O medo de engordar e o desejo constante de emagrecer podem predispor a uma imagem corporal distorcida, tal como constatado em resultados anteriores ${ }^{(19)}$ e do presente estudo. Quando distorcida, a representação da imagem corporal pode levar a comportamentos alimentares inadequados, restrição dietética e a possíveis transtornos alimentares que podem gerar consequências negativas na vida do individuo( ${ }^{(8,18-20)}$. Essas consequências incluem comorbidades psiquiátricas, transtornos ansiosos, fobia social, transtorno obsessivocompulsivo, tristeza, depressão e danos fisiopatológicos relacionados aos sistemas metabólico e endócrino(20).

O elevado percentual de insatisfação corporal encontrado entre os escolares do presente estudo está em consonância com os resultados de outros trabalhos ${ }^{(7-8,18-19)}$. Indica-se que a insatisfação com o próprio corpo manifesta-se em idades cada vez mais precoces, com indícios claros de crianças insatisfeitas com o tamanho e/ou forma corporal ${ }^{(21)}$. Essas circunstâncias podem estar associadas aos padrões imagéticos estereotipados frente às exigências sociais e culturais de peso ideal ${ }^{(6,21)}$.

Segundo os resultados da presente investigação, que convergem com os observados na literatura nacional e internacional| $\left.\right|^{(3,7-8,22)}$, tanto as crianças acima do peso quanto as eutróficas mostraram-se descontentes com sua imagem, idealizando um corpo mais magro. $O$ anseio por um corpo magro pode estar associado à valorização desse ideal, pela família e pela mídia, como sinônimo de sucesso e realização, enquanto o excesso de peso distingue-se como característica de indivíduos preguiçosos, tristes e introspectivos com possíveis prejuízos na qualidade de vida(23).

Por outro lado, o desejo por uma estrutura corporal maior entre crianças desnutridas do presente estudo assemelha-se a outro estudo(3), no qual 60\% das crianças com IMC abaixo do normal afirmaram o mesmo desejo. Isso porque, segundo a autora do estudo, esse público também sofre preconceitos em relação à forma física, semelhante às crianças obesas. Ainda, discute-se que o sexo pode ser um fator importante envolvido nessa relação, pois os meninos desejam silhuetas maiores que são valorizadas como mais musculosas e representativas do ideal masculino ${ }^{(19,22)}$.

Resultados similares à presente investigação também revelaram o sobrepeso/obesidade como características de insatisfação corporal, mostrando que à medida que aumenta o IMC aumenta a insatisfação corporal $^{(7-8,24)}$. Esse achado foi, inclusive, sintetizado em revisão sistemática da literatura mundial sobre o tema ${ }^{(22)}$. Essa condição pode ser explicada pela influência da sociedade que estigmatiza e desaprova as pessoas que estão acima do peso, bem como pela influência da mídia que, com seu poder globalizante de informação, difunde uma 
aparência idealizada e um padrão estético de magreza que é estereotipado como símbolo de beleza, saúde, sucesso e juventude ${ }^{(7,19)}$.

A forma como o individuo se vê pode ser desigual entre meninos e meninas, pois o sexo pode implicar preferências e aspirações diferentes ${ }^{(21)}$. Enquanto, segundo os padrões socioculturais e de beleza atuais, as meninas enfrentam o conflito entre o desejo pela magreza e as mudanças físicas, os meninos vivenciam uma harmonia caracterizada pela preferência por um corpo maior, forte e definido, compatível com a realidade do período de crescimento(21,25). Essa conjuntura expõe a plausibilidade da relação entre o sexo e a percepção corporal constatada não só no presente estudo(7,25).

As maiores médias de discrepância (de visão corporal, de insatisfação e de idealização) foram identificadas nas crianças mais velhas, ou seja, a maior faixa etária representando uma percepção mais distorcida de suas imagens. Esses resultados, os quais se apresentam similares aos de outros autores ${ }^{(22,25)}$, podem estar relacionados às transformações e diferenciações corporais durante o processo de maturação sexual que se inicia em idades mais avançadas e podem levar ao aumento da preocupação com a aparência física ${ }^{(22-23)}$. Observa-se que o desejo de emagrecer é significativamente superior nas crianças mais velhas, reafirmando que a magreza ganha mais importância com o passar dos anos ${ }^{(23)}$. Com o aumento da idade, os escolares experimentam mudanças físicas e psíquicas que predispõem à reorganização da imagem corporal e os tornam mais vulneráveis às influências socioculturais por um corpo ideal. Essa incongruência acaba por desencadear discrepâncias resultantes da idealização corporal|(6,25).

Apesar da ampla utilização na pesquisa, as escalas de silhuetas apresentam limitações relacionadas ao reconhecimento da imagem corporal na forma bidimensional em preto e branco, além da impossibilidade de adaptação das figuras às diferentes faixas etárias ${ }^{(23)}$. Com o intuito de minimizar esses problemas, optou-se, no presente estudo, pela utilização da escala infantil Children's Body Image Scale, pois se trata de um instrumento que, apesar de sua característica bidimensional, facilita a identificação da imagem, uma vez que usa fotografias em substituição às figuras, além de ser adaptado à faixa etária de interesse ${ }^{(12)}$. Assim, há que destacar a menor probabilidade de erro relacionado à avaliação da representação do individuo do seu corpo, com contribuição positiva para os objetivos propostos.

\section{CONCLUSÃO}

Houve prevalência expressiva de insatisfação com a imagem corporal, principalmente entre os escolares com excesso de peso que idealizam uma figura mais magra. Além disso, a visão mais distorcida do corpo nas crianças mais velhas e nos meninos alerta a necessidade de considerar o sexo, a idade e o estado nutricional da criança nas políticas públicas. Nesse sentido, destaca-se a importância de intervenções interdisciplinares das áreas de saúde e educação envolvendo a educação relacionada à imagem corporal e à nutrição por meio de uma abordagem multidimensional no ambiente escolar. Essas intervenções devem propor mudanças nos conceitos relacionados à imagem corporal que possibilitem perceber os prejuízos que a distorção perceptiva, a insatisfação corporal e a obsessão por corpos magros podem acarretar na saúde física e mental da criança. Com função preponderante na construção do sistema de cuidados, o enfermeiro pode exercer papel essencial na promoção 
da satisfação corporal das crianças prestando apoio familiar e individual com abordagem integral na saúde e bemestar.

\section{REFERÊNCIAS}

1. Pereira EF, Teixeira CS, Gattiboni BD, Bevilacqua LA, Confortin SC, Silva TR. Percepção da imagem corporal e nível socioeconômico em adolescentes: revisão sistemática. Rev Paul Pediatr [Internet]. 2011 [cited 2018 apr 17];29(3):423-9. Available from: http://dx.doi.org/10.1590/S0103-05822011000300018.

2. Palma A, Esteves AA, Assis M, Mourão L, Lüdorf SA, Vilhena LM. Imagem corporal e dependência de exercícios físicos em frequentadores de uma academia de ginástica da cidade do Rio de Janeiro. Pensar a Prática [Internet]. 2014 [cited 2018 apr 17];17(1). Available from:: https://doi.org/10.5216/rpp.v17i1.19799.

3. Vieira VA. Relação do estado nutricional e satisfação com a imagem corporal entre escolares da rede pública de ensino de São Paulo. Revista Brasileira de Obesidade, Nutrição e Emagrecimento [Internet]. 2011 [cited 2018 apr 17];5(26): 90-9. Available from: http://www.rbone.com.br/index.php/rbone/article/view/222.

4. Serafini G, Vargas IQ, Yasunaga Junior L, Tocha CP. Avaliação da imagem corporal em praticantes do método Pilates. Revista Brasileira de Reabilitação e Atividade Física [Internet]. 2014 [cited 2018 apr 17];3(1):46-51. Available from:

http://www.pilatescontemporaneo.com/wp-content/uploads/artigos/Pilates-ImagemCorporal.pdf.

5. Gonzáles C, Viebig RF. Estado nutricional de crianças praticantes de natação de uma academia paulistana. EFDeportes.com, Revista Digital [Internet]. 2012 [cited 2018 apr 17];17(173). Available from: http://www.efdeportes.com/efd173/estadonutricional-de-praticantes-de-natacao.htm.

6. Frois E, Moreira J, Stengel M. Mídias e a imagem corporal na adolescência: o corpo em discussão. Psicol em Estud [Internet]. 2011 [cited 2018 apr 17];16(1):71-7. Available from: http://dx.doi.org/10.1590/S1413-73722011000100009.

7. Neves CM, Cipriani FM, Meireles JFF, Morgado FF da R, Ferreira MEC. Imagem corporal na infância: uma revisão integrativa da literatura. Rev Paul Pediatr [Internet]. 2017 [cited 2018 apr 17];35(3):331-9. Available from: http://dx.doi.org/10.1590/19840462/;2017;35;3;00002.

8. Mancilla Medina A, Vázquez Arévalo R, Mancilla Díaz JM, Amaya Hernández A, Álvarez Rayón G. Insatisfacción corporal en niños y preadolescentes: Una revisión sistemática. Rev. Mex. de trastor. aliment [Internet]. 2012[cited 2018 apr 17]3(1):62-79. Available from: http://www.scielo.org.mx/scielo.php?script=sci arttext\&pid=S2007-15232012000100008\&lng=es.

9. Roth LR, Marin S, Rech RR, Halpern R, Pedroni JL, Sirtoli M, Cavalli A. Sintomas de Transtornos Alimentares em escolares do 6응 ano de escolas públicas municipais em uma cidade serrana do Rio Grande do Sul - Brasil. Do corpo: ciências e artes [Internet]. 2013 [cited 2018 apr 17];1(3). Available from: http://www.ucs.br/etc/revistas/index.php/docorpo/article/view/2911.

10. Lara S, Copetti J, Lanes KG, Puntel RL, Folmer V. Imagem corporal, medidas antropométricas e atividade física: ferramentas para a educação em saúde na escola. Revista Ciências \& Ideias [Internet]. 2013 [cited 2018 apr 17];4(2). Available from:

http://revistascientificas.ifrj.edu.br:8080/revista/index.php/reci/article/view/119/217.

11. World Health Organization. Physical status: the use and interpretation of anthropometry. Report of WHO Expert Committee, WHO Technical Report Series, 854. Geneva: WHO; 1995.

12. Truby H, Paxton SJ. Development of the Children's Body Image Scale. Br J Clin Psychol [Internet]. 2002 [cited 2018 apr

17];41(2):185-203. Available from: https://doi.org/10.1348/014466502163967.

13. Onis M. Development of a WHO growth reference for school-aged children and adolescents. Bull World Health Organ [Internet]. 2007 [cited 2018 apr 17];85(9):660-7. Available from: http://doi.org/10.2471/BLT.07.043497.

14. Ministério da Saúde. Orientações para a coleta e análise de dados antropométricos em serviços de saúde: Norma Técnica do Sistema de Vigilância Alimentar e Nutricional - SISVAN. Brasília: Ministério da Saúde; 2011. 76p.

15. Instituto Brasileiro de Geografia e Estatística. Pesquisa de Orçamentos Familiares 2008-2009: antropometria e estado nutricional de crianças, adolescentes e adultos no Brasil. Rio de Janeiro: Instituto Brasileiro de Geografia e Estatística; 2010. 130p. 16. Ng M, Fleming T, Robinson M, Thomson B, Graetz N, Margono C, et al. Global, regional, and national prevalence of overweight and obesity in children and adults during 1980-2013: a systematic analysis for the Global Burden of Disease Study 2013. Lancet [Internet]. 2014 [cited 2018 apr 17];384(9945):766-81. Available from: http://doi.org/10.1016/S0140-6736(14)60460-8. 17. Montarroyos ECL, Costa KRL, Fortes RC. Antropometria e sua importância na avaliação do estado nutricional de crianças escolares. Comunicação em Ciências da Saúde [Internet]. 2013 [cited 2018 apr 17];24(1):21-6. Available from:

http://doi.org/10.1016/S0140-6736(14)60460-8.

18. Bordignon S, Teodoro MLM. Relações entre percepção corporal, autoconceito e traços depressivos em crianças escolares com e sem excesso de peso. Aletheia [Internet]. 2011 [cited 2018 apr 17] (34):19-31. Available from:

http://pepsic.bvsalud.org/scielo.php?script=sci arttext\&pid=S1413-03942011000100003.

19. Leite ACB, Ferrazzi NB, Mezadri T, Höfelmann DA. Insatisfação corporal em escolares de uma cidade do Sul do Brasil. J Hum Growth Dev [Internet]. 2014 [cited 2018 apr 17];24(1):54-61. Available from: http://dx.doi.org/10.7322/ihgd.72154. 
20. Alves TCHS, Santana MLP de, Silva RCR, Pinto EJ, Assis AMO. Fatores associados a sintomas de transtornos alimentares entre escolares da rede pública da cidade do Salvador, Bahia. J Bras Psiquiatr [Internet]. 2012 [cited 2018 apr 17];61(2):55-63. Available from: http://dx.doi.org/10.1590/S0047-20852012000200001.

21. Sales RRS, Braga PHA. Percepção da imagem corporal relacionado ao índice de massa corporal de escolares 08 a 11 anos na cidade de Campo Grande, MS. EFDeportes.com, Revista Digital [Internet]. 2011 [cited 2018 apr 17];16(158). Available from: http://www.efdeportes.com/efd158/percepcao-da-imagem-corporal-de-escolares.htm.

22. Jiménez Flores $P$, Jiménez Cruz A, Bacardi Gascón M. Insatisfacción con la imagen corporal en niños y adolescentes: revisión sistemática. Nutr Hosp [Internet]. 2017 [cited 2018 apr 17]34(2):479-89. Available from: http://dx.doi.org/10.20960/nh.455. 23. Pereira ÉF, Graup S, Lopes AS, Borgatto AF, Daronco LSE. Percepção da imagem corporal de crianças e adolescentes com diferentes níveis socio-econômicos na cidade de Florianópolis, Santa Catarina, Brasil. Rev Bras Saúde Matern Infant [Internet]. 2009 [cited 2018 apr 17];9(3):253-62. Available from: http://dx.doi.org/10.1590/S1519-38292009000300004.

24. Leppers I, Tiemeier H, Swanson SA, Verhulst FC, Jaddoe VWV, Franco OH, et al. Agreement between Weight Status and Perceived Body Size and the Association with Body Size Satisfaction in Children. Obesity [Internet]. 2017 [cited 2018 apr 17];25(11):1956-64. Available from: http://doi.org/10.1002/oby.21934.

25. Pereira FN, Oliveira JR, Zöllner CC, Gambardella AMD. Body Weight Perception and Associated Factors in Students. J Hum Growth Dev [Internet]. 2013 [cited 2018 apr 17];23(2):170-6. Available from: http://dx.doi.org/10.7322/jhgd.61292. 